\title{
NUEVOS REGISTROS DE BASIDIOMYCOTA PARA JALISCO, MÉXICO
}

\author{
Olivia Rodríguez ${ }^{1,2}$, María de Jesús Herrera-Fonseca ${ }^{1}$ y Adrián Galván-Corona ${ }^{1}$ \\ ${ }^{1}$ Universidad de Guadalajara, Departamento de Botánica y Zoología, \\ Apdo. postal 1-139, 45101 Zapopan, Jalisco, México. \\ ${ }^{2}$ Autor para la correspondencia: oliviaro@cucba.udg.mx
}

\begin{abstract}
RESUMEN
Se reconocen 23 registros nuevos para la micobiota de Jalisco, de los cuales los órdenes más representativos fueron los Agaricales, Hymenochaetales y Russulales. De ellos se describen a Albatrellus caeruleoporus, Amanita jacksonii, Hydnellum ferrugineum, Hymenochaete damicornis, Pluteus salicinus, Ramaria araiospora var. araiospora y Suillus tomentosus var. discolor, y se proporciona información acerca de su importancia económica y distribución ecológica.
\end{abstract}

Palabras clave: diversidad fúngica, Jalisco, micología, San Sebastián del Oeste.

\begin{abstract}
Twenty three new records are reported for the Jalisco mycobiota, mainly pertaining to Agaricales, Hymenochaetales and Russulales. Albatrellus caeruleoporus, Amanita jacksonii, Hydnellum ferrugineum, Hymenochaete damicornis, Pluteus salicinus, Ramaria araiospora var. araiospora and Suillus tomentosus var. discolor are described, and information is provided on their economic importance and ecologic distribution.
\end{abstract}

Key words: fungal diversity, Jalisco, mycology, San Sebastián del Oeste.

\section{INTRODUCCIÓN}

En Jalisco el conocimiento de su micobiota se ha incrementado al contarse con inventarios de diferentes regiones de la entidad (Nieves, 1985; Vázquez y Guz- 
mán-Dávalos, 1988; Téllez et al., 1988; Rodríguez et al., 1994; Fierros y GuzmánDávalos, 1995; Oliva, 2000; Arroyo 2001; Herrera-Fonseca et al., 2002; Gándara, 2004), o por estudios taxonómicos de algunos géneros por Guzmán-Dávalos y Guzmán (1985, 1986, 1991), Vázquez y Guzmán-Dávalos (1991), Rodríguez et al. (1997), Rodríguez (1998), Rodríguez y Guzmán-Dávalos (2000, 2001), Guzmán-Dávalos (2003), Guzmán-Dávalos et al. (2006), Torres-Torres y Guzmán-Dávalos (2008), realizados a lo largo de casi 30 años.

Como una contribución más al conocimiento de la diversidad de especies fúngicas en Jalisco, se aportan en este trabajo 23 registros de Basidiomycota nuevos procedentes de los municipios Cabo Corrientes, Tala, Tequila, San Sebastián del Oeste, Zapopan y Zapotlán el Grande. Se describen siete taxa, por ser poco comunes, y se incluye también información acerca de la importancia económica y distribución ecológica de las mismas.

\section{MATERIALES Y MÉTODOS}

Se realizó la revisión de ejemplares de hongos depositados en los herbarios IBUG y ZT recolectados en el estado de Jalisco. El material fue estudiado de acuerdo con las técnicas convencionales en micología (Largent et al., 1977; Cifuentes et al., 1986). Para las observaciones al microscopio se efectuaron preparaciones montadas en hidróxido de potasio a 3\%; en algunos casos se utilizó solución de Melzer y rojo Congo. Se consultó la bibliografía especializada de los taxa examinados, como Breitenbach y Kränzlin (1986, 1995, 2005), Pegler (1986), Vellinga (1990), Guzmán y Ramírez-Guillén (2001) y Parmasto (2001), entre otras obras.

\section{RESULTADOS}

Se presenta un anexo con los 23 registros nuevos y sus respectivos especímenes estudiados, en el que se menciona la localidad (codificada según el Cuadro 1), así como el colector, su número, fecha y herbario. Los órdenes mejor representados fueron los Agaricales, Hymenochaetales y Russulales que corresponden al grupo de los Basidiomycota.

De acuerdo con los resultados obtenidos en este trabajo y lo encontrado por Rodríguez et al. (1994) y Herrera-Fonseca et al. (2002), se puede afirmar que en los municipios de Tequila y San Sebastián del Oeste, de los cuales procede la mayoría de 
Cuadro 1. Localidades donde fueron recolectados los ejemplares estudiados.

1. km 12-15 de la brecha Mariano Otero a San Isidro Mazatepec, Bosque La Primavera, municipio de Zapopan.

2. Bosque la Primavera, municipio de Zapopan.

3. $1 \mathrm{~km}$ camino a Los Otates - La Provincia, carretera Tomatlán - Puerto Vallarta, municipio de Cabo Corrientes.

4. km 2-6 al suroeste del Motoautódromo, Bosque La Primavera, municipio de Zapopan.

5. Ingenio de Tala (Tiradero de bagazo), municipio de Tala.

6. 50 metros de la entrada al pueblo de San Sebastián del Oeste, municipio de San Sebastián del Oeste.

7. Cerro entre la taberna de la Ermita y el Otatal, Municipio de San Sebastián del Oeste.

8. Arroyo Sonteco, km 21 San Sebastián del Oeste, La Haciendita (San Isidro), municipio de San Sebastián del Oeste.

9. El Floripondio, faldas del Nevado de Colima, camino a la Estación de Microondas Las Víboras, municipio de Zapotlán El Grande.

10. Camino Real Alto - La Bufa, 3.5-5.5 km del pueblo de San Sebastián del Oeste, municipio de San Sebastián del Oeste.

11. km 8 camino a la Estación de Microondas del Volcán de Tequila, municipio de Tequila.

12. Cerro el Tepopote, camino de ascenso a la Torre de transmisión al oeste de Nextipac, municipio de Zapopan.

13. Tierra Blanca, Segundo arroyo, Camino Real Alto - La Bufa, municipio de San Sebastián del Oeste.

14. km 18 camino a la Estación de Microondas, Volcán de Tequila, municipio de Tequila.

los ejemplares estudiados, existe una alta diversidad fúngica. Es probable que exista una riqueza similar en muchas regiones de Jalisco que aún no han sido exploradas $u$ otras en las que la recolecta de material ha sido poca.

Con base en el estudio de los taxa listados en el anexo, se presentan las observaciones y descripciones de caracteres macro y micromorfológicos de siete especies, además se incluyen fotografías para algunas de ellas.

Albatrellus caeruleoporus (Peck) Pouzar, Fig. 1.

Especie caracterizada por tener basidioma infundibuliforme, con margen ligeramente incurvado y la superficie lisa, fibrilosa o velutina, a ligeramente escamosa, con escamas pequeñas planas, de color pardo obscuro, y el resto anaranjado-gri- 
sáceo con tonos azul-grisáceos. Su himenóforo está compuesto por poros angulares, con borde lacerado, decurrentes, blanquecinos con tonos azulados, que se tornan de color anaranjado a pardo-anaranjado cuando seco. Presenta un estípite fibriloso a reticulado, más evidente hacia la base, observándose el retículo de color pardo obscuro. Sus basidiosporas son de 4-5.2 x 3.2-4.4 $\mu \mathrm{m}$, ampliamente elipsoides e inamiloides, y con un sistema hifal monomítico de hifas septadas, de pared delgada a subgruesa y sin fíbulas.

Las características macro y micromorfológicas observadas en el espécimen estudiado concuerdan con lo descrito por Valenzuela et al. (1994), quienes citaron este hongo de los estados de Durango y Nuevo León.

Albatrellus fletti es afín a $A$. caeruleoporus en virtud de los tonos azules del basidioma, pero se distingue microscópicamente por presentar hifas generativas con fíbulas (Valenzuela et al., 1994). Albatrellus caeruleoporus se reporta como medicinal (Sultana et al., 2007).

Material estudiado: JALISCO, municipio de San Sebastián del Oeste, camino a Real Alto - La Bufa, $5.5 \mathrm{~km}$ del pueblo de San Sebastián del Oeste, julio 28, 2008, E. Horak 13130 (IBUG, ZT).

Hábitat: solitario, en suelo de bosque de pino-encino.

\section{Amanita jacksonii Poumerl., Fig. 2.}

Este taxon forma parte del complejo Amanita caesarea (Scop.) Pers., se distingue macromorfológicamente por presentar un píleo convexo-umbonado, subvíscido, liso, rojo-anaranjado intenso en el centro y anaranjado hacia el margen, el cual es fuertemente estriado. Presenta basidiosporas de 9-10(-11) x 6-7(-8) $\mu \mathrm{m}$, elipsoides a ampliamente elipsoides, pocas subglobosas, con un apículo prominente, y el subhimenio formado por 2-3 células, con elementos ramosos e inflados.

De acuerdo con Guzmán y Ramírez-Guillén (2001), la especie puede confundirse con A. tullossii Guzmán \& Ram.-Guill., misma que tiene también un píleo umbonado, rojo brillante a anaranjado-rojizo; sin embargo, esta última presenta basidiosporas subglobosas a ampliamente elipsoides, y un subhimenio formado por 4-5 células globosas.

Se amplía su distribución conocida al registrarse de Jalisco; anteriormente se reportaba de los estados de Hidalgo, Michoacán, Morelos, San Luis Potosí, Veracruz (Guzmán y Ramírez-Guillén, 2001) y de Oaxaca (Garibay-Orijel et al., 2006). $\mathrm{Al}$ igual que el resto de los taxa que conforman el complejo Amanita caesarea, es considerada como comestible. 
Material estudiado: JALISCO, municipio de Cabo Corrientes, $1 \mathrm{~km}$ camino a Los Otates - La Provincia, carretera Tomatlán - Puerto Vallarta, julio 27, 2008, $O$. Rodríguez 3349 (IBUG).

Hábitat: solitario, en humus de bosque de pino-encino.

\section{Hydnellum ferrugineum (Fr.) P. Karst., Fig. 3.}

Presenta píleo turbinado-pulvinado a infundibuliforme, con la superficie zonada radialmente, con apariencia esponjosa, de color blanquecino-rosado tornándose pardorosado con tonos más pardo-negruzcos y la presencia de gotas de color rojo-sangre que exuda el basidioma en condiciones húmedas. Las basidiosporas son de 5-6.5 x 4-5 $\mu \mathrm{m}$, subglobosas a ampliamente elipsoides, tuberculadas, de color pardo claro, y las hifas de la trama himenófora son de 3-4 $\mu \mathrm{m}$ de diámetro, de pared delgada, septadas y sin fíbulas.

La caracterización macro y micromorfólogica concuerda con lo citado por Breitenbach y Kränzlin (1986), quienes describieron las basidiosporas ligeramente más pequeñas, de (4.5-)5-6 x 3.5-4.8 $\mu \mathrm{m}$; mientras que Maas Geesteranus (1975) registró esporas de (5.4-)5.8-6.3 x 3.6-4.5 $\mu \mathrm{m}$.

Hydnellum ferrugineum puede ser confundida con $H$. spongiosipes (Peck) Pouzar, ya que presenta también este último el exudado de gotas de color rojo-sangre en la superficie del píleo, sin embargo difiere microscópicamente por tener esporas ligeramente más grandes de 5.5-6.5 x 4.5-5.5 $\mu \mathrm{m}$, y por crecer en bosques de encino (Breitenbach y Kränzlin, 1986).

En México, H. ferrugineum se tiene registrada para los estados de Chiapas, Estado de México, Guerrero, Hidalgo, Morelos, Nayarit, Tlaxcala y Veracruz, de acuerdo con Cifuentes (1998). Es una especie ampliamente distribuida, reportada de Norteamérica, norte de África y Europa, considerada como probable ectomicorrizógena (Pegler et al., 1997).

Material estudiado: JALISCO, municipio de San Sebastián del Oeste, camino Real Alto - La Bufa, $5.5 \mathrm{~km}$ del pueblo de San Sebastián del Oeste, agosto 1, 2009, O. Rodríguez 3566 (IBUG).

Hábitat: gregario, en humus de bosque de pino-encino.

\section{Hymenochaete damicornis (Link) Lév.}

Se caracteriza por su basidioma estipitado, con píleo flabeliforme a espatulado y la superficie finamente fibrilosa, sulcado-zonada, de color pardo-amarillento a pardo cuero, con margen delgado, ondulado e inciso, más claro que el resto de la 
superficie del píleo. Su himenóforo es liso, de color crema-pardo-grisáceo a cremaamarillento hacia el margen. Presenta un estípite lateral, con una o varias ramificaciones, tomentoso, concoloro con la superficie del píleo y cubierto por numerosas setas. Sus basidiosporas son de 6-7 x 4-5 $\mu \mathrm{m}$, ampliamente elipsoides a elipsoides, hialinas. Se observaron setas con ápice agudo, sin incrustraciones, de color pardorojizo a pardo obscuro en el himenio, estípite y superficie del píleo.

Hymenochaete damicornis es uno de los pocos taxa del género en los que siempre se observan esporas de forma abundante, así como la presencia de setas en la superficie del píleo, himenio y estipite (Parmasto, 2001).

Hymenochaete damicornis solo se tenía reportada de los estados de Chiapas, Oaxaca y Veracruz (Marmolejo et al., 1981), aunque se conoce con una amplia distribución en el continente americano, registrada en Norteamérica (México), Centroamérica (Belice, Costa Rica, Guatemala, Honduras, Panamá y El Salvador), Caribe (Cuba, República Dominicana, Guadalupe, Jamaica, Puerto Rico y Trinidad) y para Suramérica (Argentina, Bolivia, Brasil, Colombia, Guayana Francesa, Guyana, Paraguay, Perú, Surinam y Venezuela (Parmasto, 2001). No se tiene registrado algún uso o importancia económica.

Material estudiado: JALISCO, municipio de San Sebastián del Oeste, camino Real Alto - La Bufa, $5.5 \mathrm{~km}$ del pueblo de San Sebastián del Oeste, agosto 25, 2008, M. Herrera 1223 (IBUG); municipio de Zapopan, cerro El Tepopote, agosto 13, 2010, V. Ramírez-Cruz 1359 (IBUG).

Hábitat: solitario, en suelo de bosque de pino-encino, reportada como parásito de raíces.

Pluteus salicinus (Pers.) P. Kumm., Fig. 4.

Especie que se caracteriza por el píleo pequeño de color gris-glauco a pardogrisáceo, así como por sus cistidios metuloides y una pileipellis de tipo cutis con fíbulas. $P$. salicinus generalmente muestra una coloración azul-verdosa en el basidioma al tacto o maltrato, esto debido a la presencia de psilocibina y psilocina (Saupe, 1981; Stijve y Bonnard, 1986). Microscópicamente se observan fíbulas en la base de algunas estructuras, como los queilocistidios, y cistidios metuloides tipo cervinus de pared no muy gruesa, con cuernos regularmente cortos y algo obtusos o bifurcados.

Pluteus salicinus es considerada una especie poco frecuente pero con una amplia distribución, se conoce de Europa, Norteamérica, Asia y norte de África (Breitenbach y Kränzlin, 1995); de México solo se tenía citada del estado de Hidalgo (Rodríguez y Guzmán-Dávalos, 2007). No se tiene reportado algún uso. 
Material estudiado: JALISCO, municipio de San Sebastián del Oeste, 50 metros de la entrada al pueblo de San Sebastián del Oeste, agosto 16, 2009, O. Rodríguez 3649 (IBUG).

Hábitat: gregario, lignícola en un cafetal.

Ramaria araiospora Marr \& D. E. Stuntz var. araiospora, Fig. 5.

Su basidioma es coraloide, con las ramificaciones dicotómicas hacia las puntas, las cuales tienen un ápice subagudo, con la superficie lisa, de color rosa-rojizo a rosa-coral intenso, decolorándose con la madurez, finalmente el ápice de las ramificaciones se torna de color amarillento y la base rosa-amarillenta. Al microscopio se observan basidiosporas de 8-10 × 3-4 $\mu \mathrm{m}$, cilíndricas, amarillento-oliváceas, con apículo prominente y depresión suprahilar, ornamentadas con finas verrugas que forman estrías longitudinales pero no continuas.

Por las características macro y micromorfológicas concuerda con la descripción de Castellano et al. (1999), quienes mencionaron que este taxon se distingue de la variedad rubella Marr \& D. E. Stuntz en presentar el ápice de las ramificaciones rojas cuando en la madurez.

Por la coloración rosa coral intenso a rosa pálido a crema-amarillento del basidioma, Ramaria subbotritis var. intermedia es uno de los taxa más cercanos a $R$. araiospora var. araiospora, de la cual se distingue en los ápices redondeados de sus ramificaciones (Castellano et al., 1999), así como por el tamaño más grande de las esporas (9-12 x 3.5-4(-5) $\mu \mathrm{m})$ y la forma elipsoide a subfusiforme rugosa (Bandala et al., 1987).

Ramaria araiospora var. araiospora se tiene registrada como un hongo comestible, hasta ahora solo conocido para el país del Estado de México (Aguilar-Cruz y Villegas, 2010). Se conoce también del oeste de Estados Unidos (California, Oregon, Washington) (Castellano et al., 1999).

Material estudiado: JALISCO, municipio de San Sebastián del Oeste, camino Real Alto - La Bufa, $5.5 \mathrm{~km}$ del pueblo de San Sebastián del Oeste, agosto 25, 2008, O. Rodríguez 3428 (IBUG); municipio de Tequila, $\mathrm{km} 8$ camino a la Estación de Microondas del Volcán de Tequila, septiembre 25, 2010, O. Rodríguez 3818-A (IBUG).

Hábitat: solitario a gregario, en suelo de bosque de pino-encino.

Suillus tomentosus var. discolor A. H. Sm., Thiers \& Miller

Se caracteriza por presentar el píleo convexo, con la superficie finamente fibrilosa a escamosa, de color crema a pardo-amarillento, con las escamas más 
obscuras, de color pardo, y con la superficie víscida. El himenóforo tiene poros angulares, decurrentes, de color amarillo a pardo-amarillento. El contexto se mancha de azul al exponerse. Su estípite es excéntrico, de color amarillento con tonos vináceos hacia la base, con numerosas glándulas de color pardo-rojizo, y con restos de micelio de color rosado en la base. Micromofológicamente tiene basidiospo-
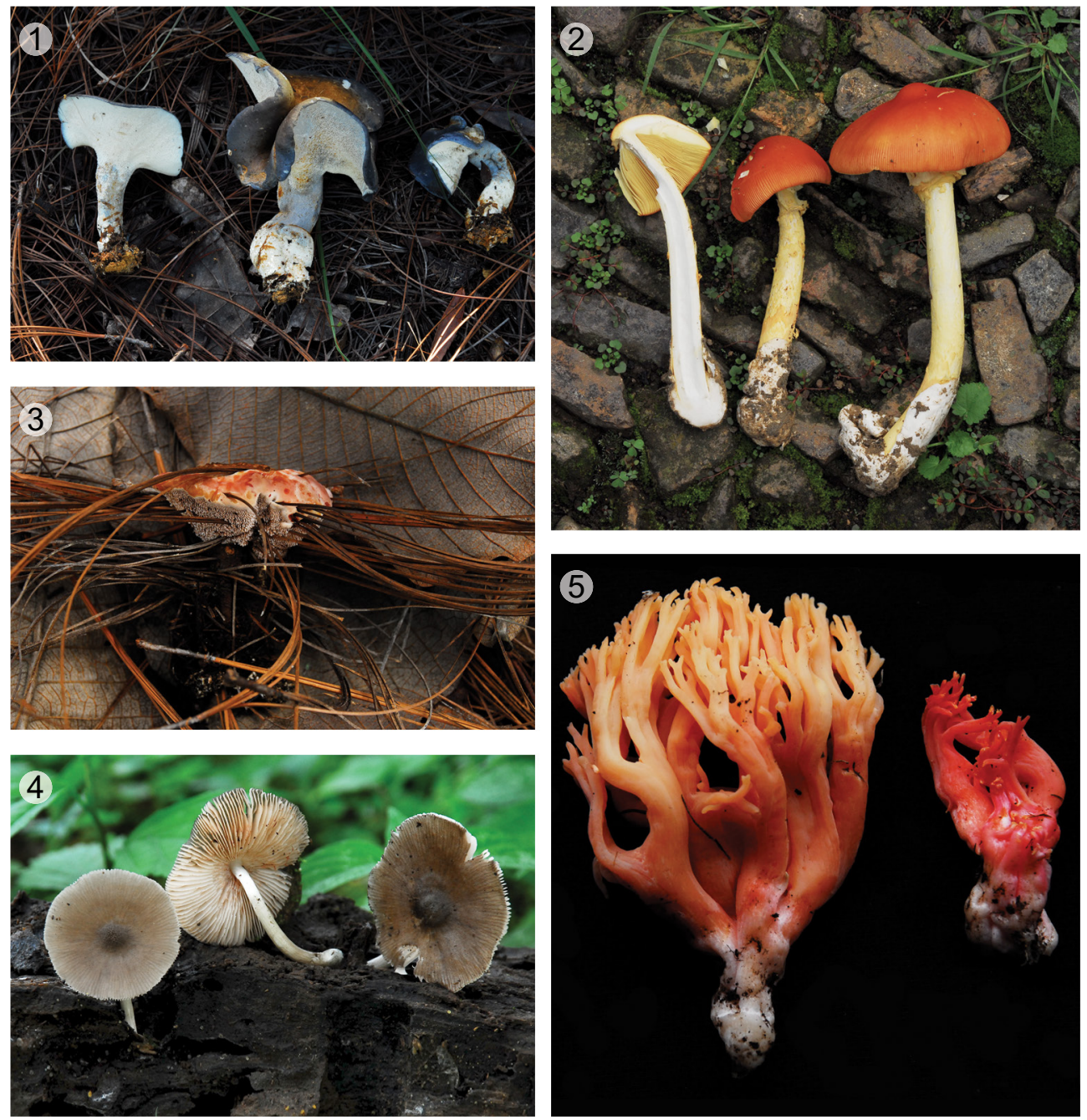

Lámina 1. Figs. 1-5. 1: Albatrellus caeruleoporus. 2: Amanita jacksonii. 3: Hydnellum ferrugineum. 4: Pluteus salicinus. 5: Ramaria araiospora var. araiospora (fotografías de Adrián Galván Corona). 
ras de 7.5-9 x 3-4 $\mu \mathrm{m}$, cilíndricas, pocas con ligera depresión suprahilar, de color pardo-oliváceo a verde-amarillento. Los queilocistidios son claviformes, con contenido granuloso, de color pardo-anaranjado, y muy abundantes. Esta variedad se distingue de la típica por presentar tonos vináceos en la base del estípite y restos de micelio rosado.

Suillus tomentosus var. discolor en México ha sido citada de los estados de Chiapas, Guerrero, Michoacán, Morelos, Nuevo León, Querétaro, Tamaulipas y Veracruz. Se reporta además del oeste de Estados Unidos, distribuida desde el estado de Idaho hasta California. Es considerada como una especie comestible y ectomicorrizógena (García, 1999).

Material estudiado: JALISCO, municipio de Zapotlán El Grande, El Floripondio, faldas del Nevado de Colima, camino a la Estación de Microondas Las Víboras, julio 25, 2008, O. Rodríguez 3309 (IBUG).

Hábitat: solitario, en suelo de bosque de pino-encino.

\section{AGRADECIMIENTOS}

Se agradece a la Dra. Laura Guzmán-Dávalos, a la M. en C. Jacqueline Reynoso Dueñas y a la Biól. María del Refugio Sánchez-Jácome por la revisión del manuscrito y sus valiosos comentarios. Los autores reconocen el apoyo de la Universidad de Guadalajara, a través de los proyectos P3E 108721 del Cuerpo Académico CA-23 y P3E 109482.

\section{LITERATURA CITADA}

Arroyo, K. 2001. Macromicetos de la Barranca del Río Santiago en Zapopan, Jalisco, con un énfasis en la conservación. Tesis profesional. Universidad Autónoma de Guadalajara. Guadalajara, Jalisco, México. 50 pp.

Bandala-Muñoz, V., L. Montoya-Bello y G. Guzmán. 1987. Nuevos registros de hongos del estado de Veracruz, III. Descripción de algunos Ascomycetes y Aphyllophorales (con nuevos registros para los estados de Hidalgo, Morelos y Tlaxcala). Rev. Mex. Mic. 3: 51-69.

Breitenbach, J. y F. Kränzlin. 1986. Fungi of Switzerland. Vol. 2. Mykologia Luzern. Lucerna, Suiza. $411 \mathrm{pp}$.

Breitenbach, J. y F. Kränzlin. 1995. Fungi of Switzerland. Vol. 4. Mykologia Luzern. Lucerna, Suiza. 368 pp. 
Breitenbach, J. y F. Kränzlin. 2005. Fungi of Switzerland. Vol. 6. Mykologia Luzern. Lucerna, Suiza. 317 pp.

Castellano, M. A., J. E. Smith, T. O’Dell, E. Cázares y S. Nugent. 1999. Handbook to strategy 1 fungal taxa from the Northwest Forest Plan. Gen. Tech. Rep. PNW-GTR-476. U.S. Department of Agriculture, Forest Service, Pacific Northwest Research Station. Portland, Oregon, USA. 195 pp.

Cifuentes, J. 1998. Distribución y algunos aspectos ecológicos de hongos hidnoides estipitados de México. Facultad de Ciencias, Universidad Nacional Autónoma de México. Base de datos SNIB-CONABIO proyecto No. H177. México, D.F., México.

Cifuentes, J., M. Villegas y L. Pérez-Ramírez. 1986. Hongos. In: Lot, A. y F. Chang (comp.). Manual de herbario. Consejo Nacional de la Flora de México. México, D.F., México. pp. 55-64.

Fierros, M. L. y L. Guzmán-Dávalos. 1995. Inventario preliminar de los hongos macroscópicos de la Sierra de Quila, Jalisco, México. Boletín IBUG 3(1-3): 129-142.

Gándara, E. 2004. Contribución al conocimiento de la micobiota del municipio de Tapalpa, Jalisco. Tesis profesional. Universidad de Guadalajara. Zapopan, Jalisco, México. 96 pp.

García, J. 1999. Estudio sobre la ecología y distribución de algunos hongos de la familia Boletaceae (Basidiomycetes, Agaricales) de México. Tesis profesional. Universidad Autónoma de Nuevo León. Linares, Nuevo León, México. 334 pp.

Garibay-Orijel, R., J. Cifuentes, A. Estrada-Torres y J. Caballero. 2006. People using macrofungal diversity in Oaxaca, Mexico. Fungal Diversity 21: 41-67.

Guzmán, G. y F. Ramírez-Guillén. 2001. The Amanita caesarea-complex. J. Cramer. Berlín, Alemania. $65 \mathrm{pp}$.

Guzmán-Dávalos, L. 2003. Type studies of Gymnopilus (Agaricales) I. Mycotaxon 86: 395423.

Guzmán-Dávalos, L. y G. Guzmán. 1985. Hongos del estado de Jalisco. V. El género Scleroderma. Rev. Mex. Mic. 1: 109-128.

Guzmán-Dávalos, L. y G. Guzmán. 1986. Hongos del estado de Jalisco, VII. El género Gymnopilus (Cortinariaceae). Rev. Mex. Mic. 2: 157-185.

Guzmán-Dávalos, L. y G. Guzmán. 1991. Additions to the genus Gymnopilus (Agaricales, Cortinariaceae) from Mexico. Mycotaxon 61: 43-56.

Guzmán-Dávalos, L., M. Herrera, B. E. Cardona y Y. Saldarriaga. 2006. Gymnopilus tuxtlensis (Basidiomycetes, Agaricales), especie tropical conocida de México y América del Sur. Acta Bot. Mex. 76: 67-75.

Herrera-Fonseca, M., L. Guzmán-Dávalos y O. Rodríguez. 2002. Contribución al conocimiento de la micobiota de la región de San Sebastián del Oeste, Jalisco, México. Acta Bot. Mex. 58: 19-50.

Largent, D., D. Johnson y R. Watling. 1977. How to identify mushrooms to genus III: Microscopic features. Mad River Press Inc. Eureka, California, USA. 148 pp.

Marmolejo, J. G., J. Castillo y G. Guzmán. 1981. Descripción de las especies de teleforáceos, poco conocidas en México. Bol. Soc. Mex. Mic. 15: 9-66.

Maas Geesteranus, R. A. 1975. The terrestrial hydnums of Europe. Verh. Kon. Ned. Akad. Wetensch. Afd. Natuurk. 65: 81-127. 
Nieves, G. 1985. Contribución al conocimiento de los macromicetos del Bosque La Primavera, Zapopan, Jalisco. Tesis profesional. Universidad de Guadalajara. Zapopan, Jalisco, México. 74 pp.

Oliva, M. 2000. Contribución al conocimiento de los hongos (macromicetos) de la Laguna de Sayula. Tesis profesional. Universidad de Guadalajara. Zapopan, Jalisco, México. $43 \mathrm{pp}$.

Parmasto, E. 2001. Hymenochaetoid fungi (Basidiomycota) of North America. Mycotaxon 79: $107-176$.

Pegler, D. N. 1986. Agaric flora of Sri Lanka. Kew Bulletin Aditional Series XII. Her Majesty's Stationery Office. Londres, UK. 519 pp.

Pegler, D. N., P. J. Roberts y B. M. Spooner. 1997. British Chanterelles and tooth-fungi. An account of the British cantharelloid and stipitate hydnoid fungi. Royal Botanic Gardens. Kew, UK. 114 pp.

Rodríguez, O. 1998. Avances en el conocimiento del género Pluteus (Fungi, Agaricales) en Nueva Galicia, México. Boletín IBUG 5(1-3): 87-93.

Rodríguez, O., M. Garza y L. Guzmán-Dávalos. 1994. Inventario preliminar de los hongos del Volcán de Tequila, estado de Jalisco, México. Rev. Mex. Mic. 10: 103-111.

Rodríguez, O., O. Vargas y L. Guzmán-Dávalos. 1997. New reports of the genus Pluteus (Agaricales) from Mexico. Mycotaxon 61: 473-480.

Rodríguez, O. y L. Guzmán-Dávalos. 2000 (como 1999). Algunas especies del género Pluteus (Pluteaceae, Agaricales) citadas en Nueva Galicia, México. Boletín IBUG 7(1-3): 61-77.

Rodríguez, O. y L. Guzmán-Dávalos. 2001. Clave dicotómica de las especies del género Pluteus Fr. (Pluteaceae) conocidas de la región de la Nueva Galicia y algunas áreas aledañas de México. Acta Bot. Mex. 57: 23-36.

Rodríguez, O. y L. Guzmán-Dávalos. 2007. Nuevos registros de Pluteus Fr. (Basidiomycetes, Agaricales, Pluteaceae) en México. Acta Bot. Mex. 80: 21-39.

Saupe, S. G. 1981. Ocurrence of psilocybin/psilocin in Pluteus salicinus (Pluteaceae). Mycologia 73: 781-784.

Stijve, T. y J. Bonnard. 1986. Psilocybine et urée dans le genre Pluteus. Mycol. Helv. 2: 123-120.

Sultana, K., G. Misbah, S. Siddiqa Firdous y R. Asghar. 2007. Hymenomycetes from Multan District. Pak. J. Bot. 39(2): 651-657.

Téllez, C., L. Guzmán-Dávalos y G. Guzmán. 1988. Contribución al conocimiento de los hongos de la Reserva de la Biosfera de la Sierra de Manantlán, Jalisco. Rev. Mex. Mic. 4: 123-130.

Torres-Torres, M. G. y L. Guzmán-Dávalos. 2008. Taxonomic status and new localities for Ganoderma ravenelii. Mycotaxon 103: 33-40.

Valenzuela R., R. Nava y J. Cifuentes. 1994. El género Albatrellus en México I. Rev. Mex. Mic. 10: 113-152.

Vázquez, L. S. y L. Guzmán-Dávalos. 1988. Algunas especies de hongos de la Barranca de Huentitán, estado de Jalisco. Rev. Mex. Mic. 4: 45-88.

Vázquez, L. S. y L. Guzmán-Dávalos. 1991. Los hongos del género Volvariella (Agaricales, Basidiomycetes) conocidos en Jalisco. Boletín IBUG 1(1): 15-22. 
Acta Botanica Mexicana 105: 45-58 (2013)

Vellinga, E. C. 1990. Pluteaceae. In: Bas, C., T. H. Kuyper, M. E. Noordeloos y E. C. Vellinga (eds.). Flora Agaricina Neerlandica. Vol. 2. A. A. Balkema. Rotterdam, Holanda. 137 pp.

Recibido en abril de 2011.

Aceptado en abril de 2013. 


\section{ANEXO}

Lista de especies que se registran por primera vez para Jalisco; se anotan los especímenes estudiados, la localidad, número de colección, fecha y herbario.

\section{BASIDIOMYCOTA}

\section{AGARICALES}

Amanitaceae

Amanita jacksonii Pomerl. O. Rodríguez 3349, loc. 1, 27-VII-2008, IBUG.

A. ponderosa Malecon \& R. Heim. L. Guzmán-Dávalos 1618, loc. 2, 11-VIII-1984, IBUG.

Psathyrellaceae

Psathyrella delineata (Peck) A.H. Sm. L. Guzmán-Dávalos 3904, loc. 1, 2-VII-1984, IBUG. Inocybaceae

Inocybe geophylla var. alba Gillet. L. Guzmán-Dávalos 3070, loc. 4, 1-VII-1986, IBUG.

Hygrophoraceae

Hygrocybe cantharellus (Schwein.) Murrill. L.M. González-Villarreal 4492, loc. 1, 28-VII1992, IBUG.

Agaricaceae

Coprinus patouillardii Quél. L. Guzmán-Dávalos 3091 loc. 5, 1-VII-1986, IBUG.

Lycoperdon molle Pers. A. Loeza 503, loc. 1, 28-VIII-1985, IBUG; J.C. Uribe 18, loc. 2, 14VII-1987, IBUG.

Pluteaceae

Pluteus salicinus (Pers.) P. Kumm. O. Rodríguez 3649, loc. 6, 16-VIII-2009, IBUG.

Marasmiaceae

Marasmius plicatulus Peck. M.R. Sánchez-Jácome 893, loc. 7, 16-IX-1995, IBUG.

Trogia buccinalis (Mont.) Pat. M. Herrera 377, loc. 8, 16-VIII-1998, IBUG.

Suillaceae

Suillus tomentosus var. discolor A.H. Sm., Thiers \& Miller. O. Rodríguez 3309, loc. 9, $25-$ VII-2008, IBUG.

CANTHARELLALES

Albatrellaceae

Albatrellus caeruleoporus (Peck) Pouzar. E. Horak 13130, loc. 10, 28-VII-2008, IBUG, ZT. GOMPHALES

Gomphaceae

Ramaria araiospora Marr \& D.E. Stuntz var. araiospora. O. Rodríguez 3428, loc. 10, $25-$

VIII-2008, IBUG; O. Rodríguez 3818-A, loc. 11, 25-IX-2010, IBUG.

HYMENOCHAETALES

Hymenochaetaceae

Hymenochaete damicornis (Link) Lév. M. Herrera 1223, loc. 10, 25-VIII-2008, IBUG; V. Ramírez-Cruz 1359, loc. 12, 29-VIII-2010, IBUG.

H. rubiginosa (Dicks.) Lév. M. Herrera 1212, loc. 10, 25-VIII-2008, IBUG.

Phylloporia spathulata (Hook.) Ryvarden. L. Guzmán-Dávalos 7402, loc. 8, 16-VIII-1998, IBUG. 
Anexo. Continuación.

\section{POLYPORALES}

Fomitopsidaceae

Fomitopsis pinicola (Sw.) P. Karst. M.R. Sánchez-Jácome 950, loc. 9, 7-IX-1996, IBUG.

Hydnaceae

Sistotrema confluens Pers. O. Rodríguez 1394, loc. 13, 16-IX-1995, IBUG.

THELEPHORALES

Bankeraceae

Hydnellum ferrugineum (Fr.) P. Karst. O. Rodríguez 3566, loc. 10, 1-VIII-2009, IBUG.

CORTICIALES

Corticiaceae

Tremellodendron candidum (Schwein.) G.F. Atk. O. Rodríguez 1336, loc. 13, 14-IX-1995, IBUG.

RUSSULALES

Russulaceae

Lactarius yazooensis Hesler \& A.H. Sm. O. Rodríguez 3712, loc. 14, 25-IX-2009, IBUG.

L. lacteolutescens Montoya, Bandala \& G. Moreno. O. Rodríguez 3709, loc. 14, 25-IX2009, IBUG.

Russula pectinatoides Peck O. Rodríguez 3711, loc. 14, 25-IX-2009, IBUG. 